\title{
Moving Ground Target Tracking in Urban Terrain Using Air/Ground Vehicles
}

Timothy McLain

Mechanical Engineering Department, Brigham Young University, mclain@byu.edu

Randal W. Beard

Department of Electrical Engineering, Brigham Young University, beard@ee.byu.edu

Mark Owen

Mechanical Engineering Department, Brigham Young University

Huili Yu

Department of Electrical and Computer Engineering, Brigham Young University

Follow this and additional works at: https://scholarsarchive.byu.edu/facpub

Part of the Mechanical Engineering Commons

\section{Original Publication Citation}

Owen, M., Yu, H., McLain, T., and Beard, R. Moving Ground Target Tracking in Urban Terrain Using Air/Ground Vehicles, IEEE Globecom 2010 Workshop on Wireless Networking for Unmanned Aerial Vehicles, pp. 1816-1820, December 2010, Miami, Florida.

\section{BYU ScholarsArchive Citation}

McLain, Timothy; Beard, Randal W.; Owen, Mark; and Yu, Huili, "Moving Ground Target Tracking in Urban Terrain Using Air/Ground Vehicles" (2010). Faculty Publications. 1912.

https://scholarsarchive.byu.edu/facpub/1912

This Conference Paper is brought to you for free and open access by BYU ScholarsArchive. It has been accepted for inclusion in Faculty Publications by an authorized administrator of BYU ScholarsArchive. For more information, please contact ellen_amatangelo@byu.edu. 


\title{
Moving Ground Target Tracking in Urban Terrain Using Air/Ground Vehicles
}

\author{
Mark Owen Huili Yu Tim McLain Randy Beard
}

\begin{abstract}
In this paper, we present a framework for tracking a moving target in urban environments using UAVs in cooperation with UGVs. The framework takes into account occlusions between the sensor and the target. The target state is modeled using the dynamic occupancy grid and the target motion model is built using a second-order Markov chain. Based on the target occupancy grid, we design the path planning algorithm to maneuver the UAV and the UGV to configurations where they can detect the target with high probability. Simulation results show the framework is successful in solving the target tracking problem in urban environments. We also build an indoor hardware platform and successfully implement preliminary tracking algorithms on the platform. Results show the platform can be further used to test more advanced tracking algorithms like the proposed tracking framework.
\end{abstract}

\section{INTRODUCTION}

Small unmanned air vehicles (UAVs) have recently found applications in the task of tracking moving targets on the ground. Many approaches to this topic have been presented in the last few years [1]-[4]. The main advantages of the target tracking using UAVs are that they have a wide field of view and can cover large areas quickly. However, sensors mounted on UAVs are unable to localize the target on the ground very accurately due to the limitations on altitude and airspeed. On the other hand, unmanned ground vehicles (UGVs) are capable of getting closer to targets and resolving their locations with greater accuracy [5]. But UGVs are slower with limited field of view. Accordingly, the complimentary strength of air and ground based sensors motivates the cooperative use of both UAVs and UGVs for target tracking.

Some approaches to the target tracking problem using both UAVs and UGVs have been proposed. Reference [6] describes an information based approach to UAV/UGV cooperative tracking. This approach works well when the targets are static, allowing the efficient use of log-likelihood filters, and when the environment is relatively free of occlusions. But it is ill-suited to tracking evasive targets in complicated urban environments. The air and ground vehicle cooperation in a probabilistic pursuit-evasion framework is considered in reference [7]. But this approach does not consider sensor data fusion, complex terrain, or planning for occluded vision. Reference [8] presents a control scheme that guides a team of

Mark Owen is a graduate research assistant in Department of Mechanical Engineering, BYU, Provo, Utah, mowen03@byu.edu

Huili Yu is a graduate research assistant in Department of Electrical and Computer Engineering, BYU, Provo, Utah, huiliyu.yhl@gmail.com

Tim McLain is a professor in Department of Mechanical Engineering, BYU, Provo, Utah, mclain@byu.edu

Randy Beard is a professor in Department of Electrical and Computer Engineering, BYU, Provo, Utah, beard@byu.edu
UGVs into a formation to effectively "corral" targets into a specific region, while a team of UAVs flies over the formation to detect targets. The approach assumes large teams of air and ground robots, and does not consider the effect of occlusions, non-navigable terrain and data fusion.

This paper presents a framework for tracking a target in urban environments using UAVs in cooperation with UGVs. Urban terrain complicates tracking problems because of the large number of occlusions and obstacles. The main contribution of the proposed framework is to take into account the locations of buildings and other objects that might occlude the line of sight between the sensors and the target. We model the target state using a dynamic occupancy grid. The target motion model is built using a second-order Markov chain and the cell occupancy of the grid is then updated using Bayesian filtering. We model the detection probabilities given the locations of the target and UAV/UGV as Gaussian functions of the distances between them when the line of sight between the sensors and the target is not occluded. We consider the effect of the occlusions by assigning the detection probabilities as zero when the line of sight between the sensors and the target is occluded. The marginal detection probabilities given the locations of the UAV/UGV are then obtained based on the detection probabilities and the target occupancy grid. To maneuver the UAV and the UGV to the configurations where they can detect the target with high probability, we design the path planning algorithms for the UAV and the UGV to find optimal paths that maximize the sum of marginal detection probabilities using T-step look-ahead policies. In addition, we build an indoor hardware platform for testing the target tracking algorithms using the UAV and the UGV. The experimental results of preliminary tracking algorithms show the platform can be used to test the proposed target tracking framework.

This paper is organized as follows. Section II describes the target state modeling and estimation using the dynamic occupancy grid. In Section III, the path planning algorithms for the UAV and the UGV are introduced. Simulation results are shown in Section IV. Section V shows the experimental platform and results of some preliminary tracking algorithms on the platform.

\section{TARGET STATE MODELING AND ESTIMATION}

In order to plan paths for the UAV/UGV team to track the target, the target state must be estimated at each time step. We model the target state using a dynamic occupancy grid, which represents changing belief about the target state. The basic idea is to represent the map as a spaced grid, 
where each grid cell has a random variable associated with it [9]. The random variable has two states, occupied and empty, which correspond to the occupancy of that cell. The dynamic occupancy grid approach utilizes Bayesian filtering to implement approximate posterior estimation for each grid cell. Bayesian filtering consists of two phases: prediction and update. In the prediction phase, the cell occupancy is predicted based on its posterior probability at the previous time step and the target motion model. We construct the target motion model using the second-order Markov chain. The model assumes that the target most likely continues moving in the direction that it has been moving. In the update phase, the predicted occupancy probability is updated by the measurements. Let $x_{k}^{t}$ represent the binary state variable associated with a grid cell $C$ at time step $k$ and let $z_{k}$ represent the random variable associated with the measurement. Let $\bar{P}\left(x_{k}^{t}\right)$ represent the probability obtained by the prediction step and let $P\left(z_{k} \mid x_{k}^{t}\right)$ represent the likelihood probability. Using Bayes rule, the posterior probability of the cell $C$ at time step $k$ is given by $P\left(x_{k}^{t} \mid z_{k}\right)=\eta P\left(z_{k} \mid x_{k}^{t}\right) \bar{P}\left(x_{k}^{t}\right)$, where $\eta$ is a normalization term.

\section{Path Planning Algorithms}

Given the target occupancy grid, we design the path planning algorithms for the UAV/UGV for tracking the target. The objective of the algorithms is to generate paths for the UAV/UGV so that they can detect the target with the high probability along the paths.

\section{A. Detection probability and marginal detection probability}

Before introducing the planning algorithm for the UAV target tracking, we define the notions of the detection probability and marginal detection probability. Let $x_{k}^{a}$ and $x_{k}^{g}$ represent the random variables associated with the locations of the UAV and the UGV at time step $k$. Let $z_{k}^{a}$ and $z_{k}^{g}$ represent the measurement obtained by the sensors on the UAV and the UGV. Given $x_{k}^{a}$ and the target location $x_{k}^{t}$, when the line of sight between the sensor and the target is not occluded, we model the detection probability for the UAV as a Gaussian function of the distance between the UAV and the target given by

$$
P\left(D \mid x_{k}^{a}, x_{k}^{t}\right)=\eta \exp \left(-\frac{1}{2}\left(x_{k}^{a}-x_{k}^{t}\right)^{T} \Sigma^{-1}\left(x_{k}^{a}-x_{k}^{t}\right)\right),
$$

where $D$ is a binary random variable indicating whether the detection made by the UAV is correct or false and $\eta$ is normalization term. When the line of sight is occluded, we assign the probability as zero. Figure 1 shows an example of the detection probability. In this example, the target location is held constant at $(0,0)$, while the vehicle location is varied in one meter increments on both axes. The detection probability given by Eq. (1) can be precomputed for each pair of the discretized UAV and target locations. The marginal detection probability given the UAV location at time step $k$ is given by

$$
P\left(D \mid x_{k}^{a}\right)=\sum_{x_{k}^{t}} P\left(D \mid x_{k}^{a}, x_{k}^{t}\right) P\left(x_{k}^{t}\right) .
$$

Similarly, the detection probability given the UGV location

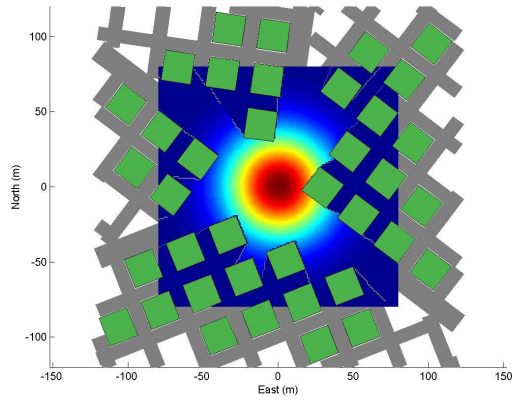

Fig. 1. The detection probability when buildings occlude the line of sight vector.

$x_{k}^{g}$ and the target location $x_{k}^{t}$ for the UGV is given by

$$
P\left(D \mid x_{k}^{g}, x_{k}^{t}\right)=\eta \exp \left(-\frac{1}{2}\left(x_{k}^{g}-x_{k}^{t}\right)^{T} \Sigma^{-1}\left(x_{k}^{g}-x_{k}^{t}\right)\right) .
$$

The marginal detection probability given the UGV location is then given by

$$
P\left(D \mid x_{k}^{g}\right)=\sum_{x_{k}^{t}} P\left(D \mid x_{k}^{g}, x_{k}^{t}\right) P\left(x_{k}^{t}\right) .
$$

\section{B. Path planning algorithm for the UAV target tracking}

The path planning algorithm for the UAV is designed to generate a T-step look-ahead path maximizing the sum of the marginal detection probability along the path given by

$$
J_{a}=\sum_{j=0}^{T} P\left(D \mid x_{k+j \Delta k}^{a}\right) .
$$

Since different roll commands generate different paths for the UAV, we parameterize the paths by roll angles and choose the optimal path maximizing the reward function $J_{a}$ among the paths. We achieve this by recursively searching a Tstep look-ahead planning horizon tree. Each node in the tree represents the UAV configuration at a certain step look-ahead planning horizon and it has multiple children, each of which represents the resulting configuration at the next step for a certain roll command. Each path from the root to a leaf represents the resulting T-step look-ahead path for the UAV when a certain set of roll commands are applied. Figure 2 shows how the UAV path planning algorithm works using a two-step look-ahead planning horizon tree, where each node has three children at the next step corresponding to three different roll commands. At time step $k$, the UAV is at the configuration $x_{k}^{a}$ and the path $\left\{x_{k}^{a}, x_{k+\Delta k}^{a}, x_{k+2 \Delta k}^{a}\right\}$ has been found, as shown in Fig. 2(a). The UAV is maneuvered to $x_{k+\Delta k}^{a}$. During the period, $x_{k+\Delta k}^{a}$ is first used as the tree root and the branches whose root is not at $x_{k+\Delta k}^{a}$ are removed. The tree is then extended by one step horizon and the new tree is searched to find a new path $\left\{x_{k+\Delta k}^{a}, x_{k+2 \Delta k}^{a}, x_{k+3 \Delta k}^{a}\right\}$. Searching the tree and finding a path can be solved efficiently using dynamic programming [10]. Once the UAV reaches $x_{k+\Delta k}^{a}$, the new path $\left\{x_{k+\Delta k}^{a}, x_{k+2 \Delta k}^{a}, x_{k+3 \Delta k}^{a}\right\}$ has been found, as shown in Fig. 2(b). The process is repeated so that the UAV is always 
maneuvered to the configurations with high marginal detection probabilities. The path planning algorithm can be implemented in real time since the UAV can always be maneuvered to the next tree node once it reaches a tree node.

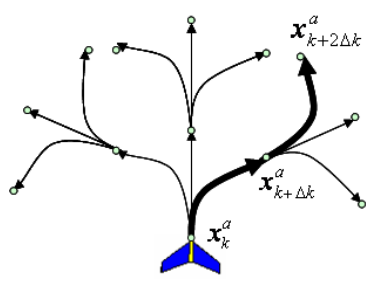

(a)

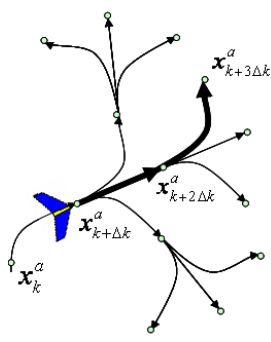

(b)
Fig. 2. The two-step look-ahead path planning tree for the UAV

\section{Path planning algorithm for the UGV target tracking}

The path planning algorithm for the UGV is designed to generate a T-step look-ahead path maximizing the reward function

$$
J_{g}=\sum_{j=0}^{T} P\left(D \mid x_{k+j \Delta k}^{g}\right) .
$$

Since the UGV can only move along the roads, we decompose the roads into cells and construct a graph using those cells. The detection probability given by Eq. (3) can then be precomputed for each pair of the locations of the UGV and the target. Based on the graph, the T-step lookahead planning horizon tree is constructed. The connectivity of the graph determines the extension of the tree. Figure 3 shows how the UGV path planning works using a two-step look-ahead planning horizon tree, where the circles represent the nodes. At time step $k$, the UGV is at the configuration $x_{k}^{g}$ and the path $\left\{x_{k}^{g}, x_{k+\Delta k}^{g}, x_{k+2 \Delta k}^{g}\right\}$ has been found as shown in Fig. 3(a). The UGV is maneuvered to $x_{k+\Delta k}^{g}$. During the period, the branches whose root is not at $x_{k+\Delta k}^{g}$ are removed. The tree is then extended by one step horizon and the new tree is searched to find a new path $\left\{x_{k+\Delta k}^{g}, x_{k+2 \Delta k}^{g}, x_{k+3 \Delta k}^{g}\right\}$ using dynamic programming. Once the UGV reaches $x_{k+\Delta k}^{g}$, the new path $\left\{x_{k+\Delta k}^{g}, x_{k+2 \Delta k}^{g}, x_{k+3 \Delta k}^{g}\right\}$ has been found, as shown in Fig. 3(b).

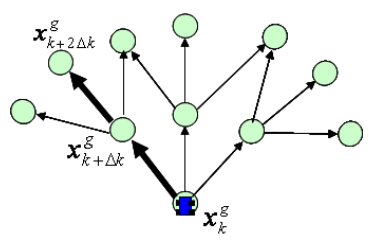

(a)

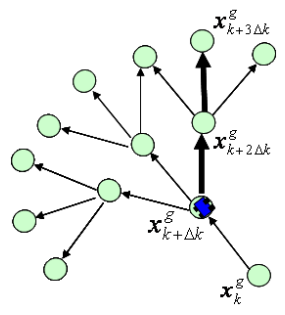

(b)
Fig. 3. The two-step look-ahead path planning tree for the UGV.

\section{Simulation}

The algorithm was tested using a simulation environment developed in MATLAB/SIMULINK, as shown in Fig. 4, where green blocks represent the buildings. A $49 \times 49$ occupancy grid, where the size of each cell is $5 m \times 5 m$, is used to model the target state. The simulator uses six state navigation equations of the aircraft and uses four state navigation equations of the ground vehicle. The four-step and six-step lookahead horizon paths are planned for the UAV and the UGV respectively. In the simulation, the target is initially placed at North-East coordinate $(75 \mathrm{~m}, 75 \mathrm{~m})$ and it will move among the waypoints $(75 \mathrm{~m}, 75 \mathrm{~m}),(75 \mathrm{~m},-75 \mathrm{~m}),(-75 \mathrm{~m},-75 \mathrm{~m})$ and $(-$ $75 \mathrm{~m}, 75 \mathrm{~m})$ in turn. The motion model of the target is not known by the UAV and the UGV.

Figure 4 shows the snapshots of target occupancy grid and the paths of the UAV and the UGV for tracking the target at different time steps. The algorithm assumes the target is initially located at the origin. It then updates the target occupancy grid using Bayesian filtering and plans the corresponding paths based on the target occupancy grid such that the reward functions $J_{a}$ and $J_{g}$ are maximized. By doing so, the UAV and the UGV can eventually track the target at time $t=48 s$, as shown in Fig. 4(c). Figure 5 shows the results of the target tracking for the UAV and the UGV once the target has been tracked.
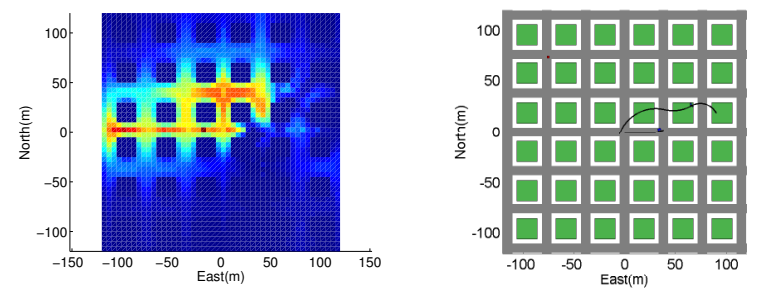

(a) $t=25 \mathrm{~s}$
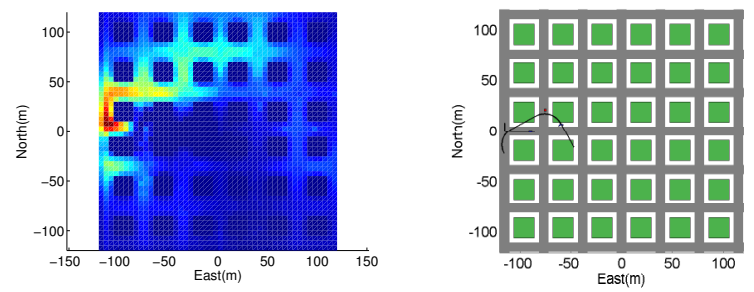

(b) $t=35 \mathrm{~s}$
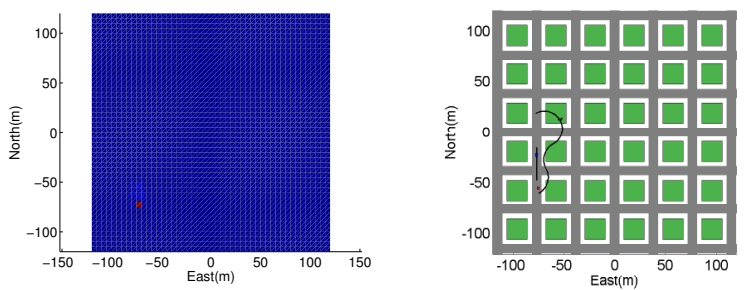

(c) $\mathrm{t}=48 \mathrm{~s}$

Fig. 4. The snapshots of target occupancy grid and the paths of the UAV and the UGV.

\section{EXPERIMENTAL PLATFORM AND RESULTS}

\section{A. Experimental platform}

Since FAA regulations make it difficult to perform UAV flight tests outdoors, an indoor model of an urban environment 


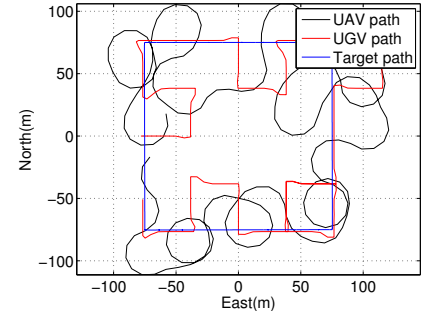

Fig. 5. The results of the target tracking for the UAV and the UGV.

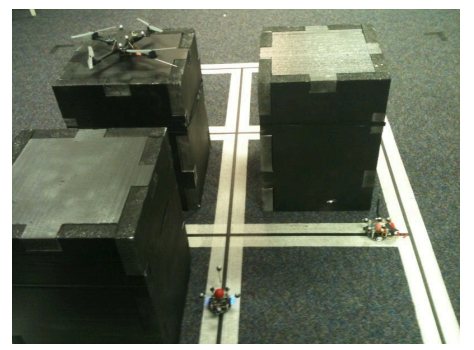

Fig. 6. The city environment

was used for testing. While indoor testing is convenient, it poses other technical challenges. Specifically, GPS cannot be used for state estimation indoors and even small fixed-wing UAVs need a lot of space for normal flight.

To reduce the amount of space needed for flight tests a miniaturized urban model measuring 4 meters by 3 meters was developed. A rotorcraft was used in place of a fixedwing vehicle. A constant-velocity controller was developed to simulate fixed-wing flight. In order to minimize the challenges of ground navigation line-following robots were used as the pursuit and target ground vehicles. For state estimation a 3D camera positioning system from Motion Analysis was used.

In order to simulate an urban environment we constructed a scaled down city. The overall size of the city was constrained by the size of the functional volume of the 3D camera positioning system. Buildings were fabricated and placed in the center of 5 of the 8 city blocks in a U-shape. Roads suitable for the line-following robots were produced by placing black tape on wide masking tape. See Figure 6.

As mentioned previously, a camera-based 3D positioning system from Motion Analysis was used as a replacement for GPS. The particular setup for this experiment used 8 cameras situated around the edge of a 9 meter by 5 meter room. The usable volume (ie. the volume in which two or more cameras can always see an object) is $4 m \times 3 m \times 1.5 m$. Each camera in the positioning system uses an array of near-infrared LEDs to illuminate the room. Synchronized images from all cameras are returned to a central computer. A set of reflective points can be attached to a rigid body in a known configuration called a "prop." The quadrotor, ground and target robot props can be seen in Figure 7. The positioning software provided with the cameras, "Cortex," fits props to image data received from the cameras. If at least two cameras detect the same reflective object the 3D position and orientation of the object is computed. Prop states (position, orientation) can then be

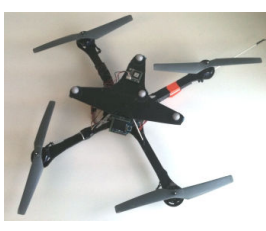

(a) Quadrotor

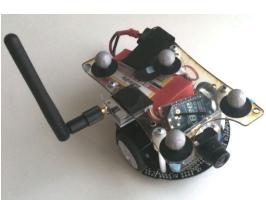

(b) Ground Robot (c) Target

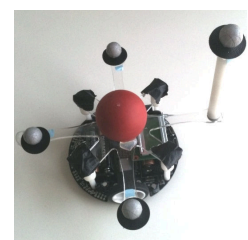

Fig. 7. Modified testbed hardware with Cortex prop sets

streamed over a TCP/IP connection at up to $200 \mathrm{~Hz}$.

We selected the Hummingbird quadrotor from Ascending Technologies for testing as shown in Fig. 7(a). This quadrotor comes equipped with an autopilot that can be configured to run either rate-hold or angle-hold control loops at $1000 \mathrm{~Hz}$. For communication, a custom board was designed to connect XBee radios from Digi directly to the Hummingbird autopilot. On the ground, a matching XBee radio was connected to a desktop computer that ran control algorithms.

We used a Pololu $3 \pi$ line-following robot to represent the ground vehicle as shown in Fig. 7(b). In order to facilitate communication between the robot and the path planner we setup an XBee radio with the device and had a corresponding radio connected to the computer in the same fashion as the quadrotor. The device was programmed to follow the line until it sensed an intersection at which point it would turn right, left or continue straight based on the most recent commands it had received. Buffered commands were sent to the UGV from the path planner with the next four desired turns and were updated each time the target robot passed an intersection.

The robot used as a target was also a Pololu $3 \pi$ linefollowing robot as shown in Fig. 7(c). It simulated a randomly wandering ground target. The robot was programmed to follow the line it was on and randomly turn at intersections. A red ball was mounted on top of the robot to facilitate visual target identification.

Digital cameras and transmitters were outfitted to both tracking vehicles so video could be sent to a central computer for processing. The intent is to use the collaborative video feeds to estimate the states of the target. These estimates will soon be used to continually update a target occupancy probability map.

A central application was written to receive all robot states from Cortex, receive high-level control commands from a Matlab path planner, run velocity and heading control loops for the quadrotor, and send commands to the quadrotor and ground robot. Figure 8 shows a diagram of the complete system architecture used for testing.

\section{B. Results}

The UAV path planner is very rudimentary in its current configuration. Based on truth data from Cortex the UAV was commanded to orbit around the target's current position with a fixed radius and velocity. This was a preliminary path planner for use during system integration. The results of more 


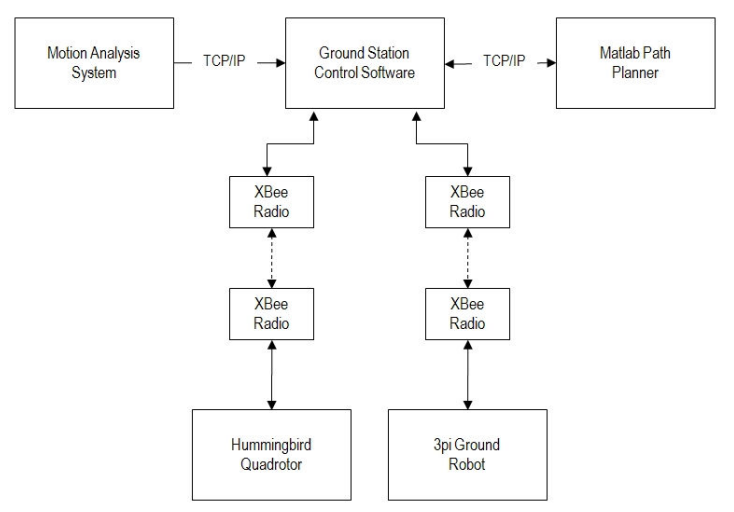

Fig. 8. Flowchart for experimental setup.

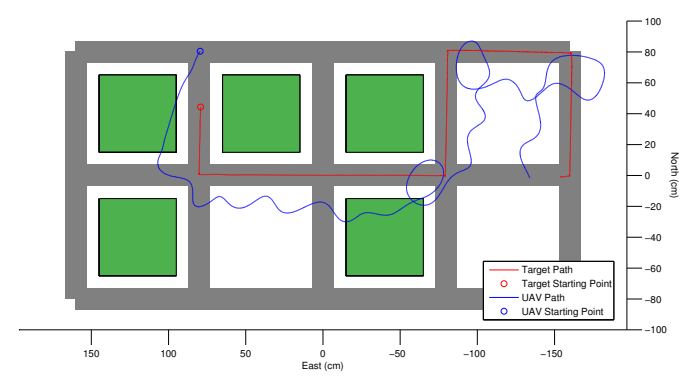

Fig. 9. Experimental Results: UAV Target Tracking.

advanced path planners such as those discussed in this paper are forthcoming.

Visual inspection of the quadrotor path confirms the validity of using it to simulate a fixed-wing aircraft (see Figure 9). Due to the difference in vehicle speeds, as the quadrotor attempts to fly orbits around the target it is apparent that the rotorcraft flies a path characteristic of a fixed-wing aircraft. The aircraft weaves alongside the target location and occasionally flies a complete orbit around the target such as when the vehicle slows to make a turn.

The ground robot path planner is based on a graph representation of the city. The city setup specifications are loaded from an $\mathrm{xml}$ file of the format produced by a program called CityMaker. After they are loaded a list is made of all permutations of the four possible turns starting from each intersection. With knowledge of the UGV and target states the algorithm finds the optimal path to the intersection behind the target over a four-step look-ahead horizon using dynamic programming. Those turns are then sent to the UGV and stored as the next four turn commands.

The experimental results show that the ground robot effectively navigates behind (the intersection behind) the target (see Figure 10). In this experiment the UGV had a greater maximum speed than the ground robot in order to allow it to close the distance between the two vehicles.

\section{CONCLUSIONS}

We present a framework for tracking a moving target in urban environments using both UAVs and UGVs. Occlusions are taken into account in the framework. We use the dynamic occupancy grid to model the target state. The occupancy

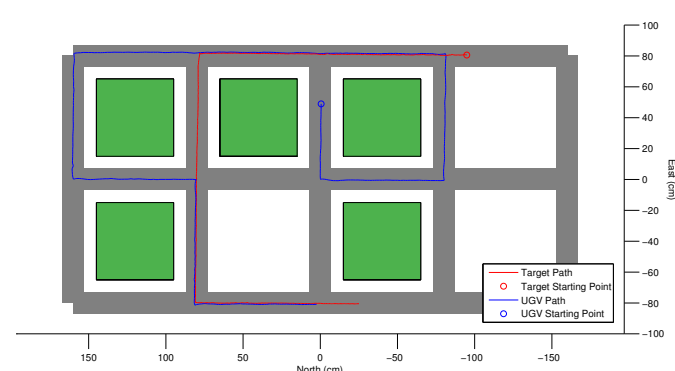

Fig. 10. Experimental Results: UGV Target Tracking.

grid is updated using Bayesian filtering. We design the path planning algorithms to maneuver the UAV and the UGV to configurations where they can detect the target with high probability. Based on simulation results, the framework is successful in solving the target tracking problem in urban environments. In addition, an indoor hardware platform is built and preliminary tracking algorithms are implemented on the platform.

In the paper, the paths are planned based on the individual UAV/UGV detection probability. In the future, we will design the planning algorithm for the UAV and the UGV to track the target cooperatively using their joint detection probability. We will consider the communication constraints between the UAV and the UGV. We will conduct the Monte Carlo simulation and perform a statistic analysis of the algorithm. We will also implement the proposed tracking framework on the platform.

\section{ACKNOWLEDGEMENT}

This research was supported by OSD and AFRL under contract FA8650-08-C-1411 and was partially supported under U.S. Army ARDEC contract W15QKN-09-C-0173 with BYU serving as a subcontractor to Procerus Technologies.

\section{REFERENCES}

[1] J. Kim and Y. Kim, "Moving target tracking in dense obstacle areas using UAVs," in Proc. 17th IFAC World Congress, Seoul, Korea, 2008.

[2] Z. Tang and U. Ozguner, "Sensor fusion for target track maintenance with multiple UAVs based on Bayesian filtering method and hospitability map," in Proc. 42nd IEEE Conference on Decision and Control, vol. 1, 9-12 Dec. 2003, pp. 19-24.

[3] U. Zengin and A. Dogan, "Real-time target tracking for autonomous UAVs in adversarial environments: A gradient search algorithm," vol. 23, no. 2, pp. 294-307, April 2007.

[4] S. Kanchanavally, R. Ordonez, and J. Layne, "Mobile target tracking by networked uninhabited autonomous vehicles via hospitability maps," in Proc. American Control Conference, vol. 6, 2004, pp. 5570-5575.

[5] B. Grocholsky, R. Swaminathan, J. Keller, V. Kumar, and G. Pappas, "Information driven coordinated air-ground proactive sensing," in Proc. IEEE International Conference on Robotics and Automation ICRA 2005, 18-22 April 2005, pp. 2211-2216.

[6] B. Grocholsky, J. Keller, V. Kumar, and G. Pappas, "Cooperative air and ground surveillance," IEEE Robotics \& Automation Magazine, vol. 13, no. 3, pp. 16-25, Sept. 2006.

[7] R. Vidal, O. Shakernia, H. J. Kim, D. H. Shim, and S. Sastry, "Probabilistic pursuit-evasion games: theory, implementation, and experimental evaluation," vol. 18, no. 5, pp. 662-669, Oct. 2002.

[8] H. G. Tanner, "Switched UAV-UGV cooperation scheme for target detection," in Proc. IEEE International Conference on Robotics and Automation, 10-14 April 2007, pp. 3457-3462.

[9] S. Thrun, W. Burgard, and D. Fox, Probabilistic Robotics. The M.I.T. Press: Cambridge, Massachusetts, 2005.

[10] F. L. Lewis, Optimal Control. New York: John Wiley \& Sons, 1986. 